hep-th/0411174

\title{
Black Hole Non-Formation in the Matrix Model
}

\author{
Joanna L. Karczmarek, Juan Maldacena and Andrew Strominger
}

\begin{abstract}
The leading classical low-energy effective actions for two-dimensional string theories have solutions describing the gravitational collapse of shells of matter into a black hole. It is shown that string loop corrections can be made arbitrarily small up to the horizon, but $\alpha^{\prime}$ corrections cannot. The matrix model is used to show that typical collapsing shells do not form black holes in the full string theory. Rather, they backscatter out to infinity just before the horizon forms. The matrix model is also used to show that the naively expected particle production induced by the collapsing shell vanishes to leading order. This agrees with the string theory computation. From the point of view of the effective low energy field theory this result is surprising and involves a delicate cancellation between various terms.
\end{abstract}




\section{Contents}

1. Introduction . . . . . . . . . . . . . . . . . . . . . . . . . . . . . 1

2. Spacetime gravitational collapse . . . . . . . . . . . . . . . . . . . . 3

2.1. The tachyon . . . . . . . . . . . . . . . . . . . . . . . . . . . . . . . . 4

2.2. The axion . . . . . . . . . . . . . . . . . . . . . . . . . . . . . . . . . 4

2.3. Corrections . . . . . . . . . . . . . . . . . . . . . . . . . . . . . . . . 7

3. Matrix model picture . . . . . . . . . . . . . . . . . . . . . . . . . . . . . . 9

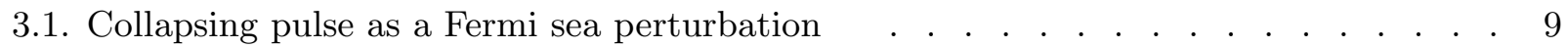

3.2. Outgoing radiation on $\mathcal{I}^{+}$. . . . . . . . . . . . . . . . . . . . . . . . . 12

4. Particle Production in the Spacetime Picture . . . . . . . . . . . . . . . . . . . 14

4.1. Particle creation by an infalling pulse . . . . . . . . . . . . . . . . . . . . . 14

4.2. A time dependent mass . . . . . . . . . . . . . . . . . . . . . . . . . . . 15

4.3. The Quartic Interaction . . . . . . . . . . . . . . . . . . . . . . . . . . . 17

4.4. Particle production in string theory . . . . . . . . . . . . . . . . . . . . . 17

5. Particle Production in the Matrix Model . . . . . . . . . . . . . . . . . . . . . 21

5.1. Basic formulae . . . . . . . . . . . . . . . . . . . . . . . . . . . . . . . 21

5.2. Single fermion reflection . . . . . . . . . . . . . . . . . . . . . . 22

5.3. One point functions . . . . . . . . . . . . . . . . . . . . . . . . . . . . 23

5.4. Axion two point functions . . . . . . . . . . . . . . . . . . . . . . . . . . 26

5.5 . Tachyon two point function . . . . . . . . . . . . . . . . . . . . . . . . . 28

6. Scattering amplitudes for very energetic states . . . . . . . . . . . . . . . . 28

7. Discussion . . . . . . . . . . . . . . . . . . . . . . . . . . . . . . . . . . 30

7.1. The definition of time . . . . . . . . . . . . . . . . . . . . . . . . . . . . 31

7.2. The non-singlet sector . . . . . . . . . . . . . . . . . . . . . . . . . . . 32

7.3. $S L(2)_{k} / U(1)=$ black hole for small $k$ ? . . . . . . . . . . . . . . . . . . . . . 32

7.4. Conclusions . . . . . . . . . . . . . . . . . . . . . . . . . . . . . . . . 34

\section{Introduction}

Despite much recent progress, a number of interesting questions concerning twodimensional string theories and their matrix model solutions remain unanswered. Among them is the seemingly simple question of whether or not the theories contain black holes. If they do, we would like to understand the quantum behavior of the black holes. If they do not, we would like to understand why not.

The continuum $2 \mathrm{~d}$ string theory has an exact classical solution given by an $S L(2, R)_{k} / U(1)$ worldsheet CFT [1]. For large $k$ this CFT has an unambiguous interpretation as a spacetime black hole [2, 3, 1 1]. Moreover, for the small values of $k$ relevant

1 More precisely for large $k$ it is a factor of the CFT for the near extremal black fivebrane in critical string theory 泗. For small $k$, which is relevant to the present work, the black hole interpretation is less clear, as will be discussed in the concluding section. 
to $2 \mathrm{~d}$ string theory, an exact matrix model (significantly involving the non-singlet sector) which generalizes this classical solution to the full quantum theory is known [5]. On the face of it these facts suggest that black holes indeed arise in $2 \mathrm{~d}$ string theories much as in their higher dimensional cousins. Furthermore, the computations of [5] show not only that the $S L(2) / U(1)$ black hole arises in the non-singlet sector of the matrix model but also that there is a wider family of black holes than we would have suspected from the low energy effective action, since one can vary the temperature.

On the other hand, so far there has been no clear indications of black hole formation in any matrix model scattering amplitudes [6], [7] (see [8] for an early discussion). There is also no sign of a large degeneracy of states which would be required for a microscopic accounting of black hole entropy, or the phase transition signaling black hole formation. Finally, the quintessentially thermal nature of black holes seems at odds with the integrability of the matrix model [9].

Since all of these facts involve purely the singlet sector of the matrix model, all that is known so far is consistent with the black holes arising only in the non-singlet sector. Since the non-singlet sector is much harder to analyze in the Lorentzian context than the singlet sector, we would like to make sure we are not missing some black hole configurations that arise in the singlet sector.

In this paper we address this problem by trying to make black holes by collapsing the tachyonic or axionic matter. It is shown that the low-energy effective action indeed has solutions in which such collapse occurs in a region where string loop corrections can be made arbitrarily small. This suggests that the problem of black hole formation is a question for classical string theory or equivalently worldsheet conformal field theory. On the other hand, $\alpha^{\prime}$ corrections can never be controlled, so a full classical string analysis is needed. Using the matrix model, we show that a typical collapsing pulse does not form a black hole. Rather it bounces off of the would-be event horizon just before the black hole is about to form, and the matter is backscattered back out to infinity!

We further analyze the problem of particle creation by the collapsing shell. Naively, one expects a rising tail of outgoing Hawking radiation even before a black hole forms. A matrix model computation is used to show that the leading term (at early times) of tachyon particle production on $\mathcal{I}^{+}$vanishes. A consistent picture emerges from a careful analysis of the spacetime effective action, which reveals several sources of particle production in addition to the Hawking radiation. For appropriate numerical values of the effective action coefficients, these different contributions can cancel, in agreement with the matrix 
model computation. We also perform the exact (in $\alpha^{\prime}$ ) string theory scattering amplitude computation and show that the amplitude vanishes here (this is the bulk $(2,2)$ amplitude studied in [10,11].

Our results are certainly consistent with the idea that there are no black holes in the singlet sector of the matrix model. At the same time, our investigations have uncovered more curious behavior indicating there is much we do not understand about the matrix model. As such we feel it is too early to jump to conclusions. Further discussion can be found at the end of this paper.

This paper is organized as follows: In section 2 we describe the collapse of a matter shell in the 2 dimensional effective theory for $0 \mathrm{~B}$ string and discover that while other corrections are under control, the curvature on the would-be horizon is of order one and thus the effective description breaks down. In section 3 we describe the same pulse in matrix theory and discover that most of the energy does in fact escape to infinity at parametrically the same retarded time at which the horizon would have formed. In section 4 we switch gears and begin to study early time particle creation in the collapsing pulse. We consider various sources of early time outgoing stress energy for both the tachyon and the axion in effective theory. In 4.4 we study the related string theory S-matrix element. In section 5, we study this outgoing stress energy in the matrix model at $\mu=0$ using the free fermion formulation. Finally, in section 6, particle creation due to a very energetic incoming state is considered, with implications for possible thermality of the outgoing state. We close with discussion in section 7 .

Related independent work has recently appeared in [12]. Previous work on particle creation in the matrix model includes 13,16].

\section{Spacetime gravitational collapse}

The leading bosonic terms of the low-energy effective action for type 0B string theory are

$$
S_{e f f}=\frac{1}{2 \pi} \int d^{2} x \sqrt{-g}\left(e^{-2 \Phi}\left(16+R+4(\nabla \Phi)^{2}-\frac{1}{2}(\nabla T)^{2}+2 T^{2}\right)-\frac{1}{2}(\nabla C)^{2}+\ldots . .\right)
$$

In this expression $\alpha^{\prime}=\frac{1}{2}, T$ is the NS-NS sector "tachyon" and $C$ is the RR axion. The position-dependent string coupling is given by the dilaton

$$
g_{s}=e^{\Phi}
$$


The corrections to (2.1) involve higher derivatives, more powers of $g_{s}$ and/or higher nonlinear dependence on $T$. To all orders in perturbation theory there is a RR shift symmetry which prohibits the axion $C$ from appearing without a derivative. The classical terms in the action all scale like $\lambda^{2}$ under $\Phi \rightarrow \Phi-\ln \lambda, \quad C \rightarrow \lambda C$, so that the classical limit is

$$
C \sim e^{-\Phi} \sim \frac{1}{g_{s}} \rightarrow \infty
$$

In this section we study black hole formation using the effective action (2.1), and also discuss when and how the approximation (2.1) to the exact theory becomes unreliable.

\subsection{The tachyon}

The question of whether or not gravitational tachyon collapse occurs at $c=1$ was discussed in the early days of the bosonic matrix model, which does not have the axion. A naive argument that there is no reliable approximation in which the tachyon field can be seen to form an event horizon goes as follows. In order for an event horizon to form, the metric and or dilaton must be of order one. The equations of motion following from (2.1) allow this to happen only if the tachyon $T$ is also of order one. However if the tachyon is of order one, its self interactions are important, and (2.1) can not be trusted. Hence one cannot be sure that gravitational collapse of tachyons can really occur.

This argument is a bit too fast because we should also consider the possibility of making $T$ small by spreading out the pulse. However the problem of tachyon collapse is still difficult to analyze in part because of the complicated tachyon-dilaton interactions present in (2.1), as well as the fact that the stress tensor is not positive definite even at leading order. We therefore turn to the problem of axion collapse which turns out to be much simpler.

\subsection{The axion}

Setting the tachyon to zero and scaling the axion as in (2.3), the action (2.1) becomes precisely the classical CGHS [17] action coupling dilaton gravity to conformal matter. 2 It is convenient to choose conformal gauge $g_{\mu \nu}=e^{2 \rho} \eta_{\mu \nu}$ or, in light-cone coordinates,

$$
d s^{2}=-e^{2 \rho} d x^{+} d x^{-}
$$

2 This is not exactly a coincidence, since the CGHS theory was derived from the linear dilaton theory in the NS fivebrane throat. 
We then have $R=8 e^{-2 \rho} \partial_{+} \partial_{-} \rho$ and the equations of motion become

$$
\begin{array}{ll}
\Phi: & e^{-2(\Phi+\rho)}\left[-4 \partial_{+} \partial_{-} \Phi+4 \partial_{+} \Phi \partial_{-} \Phi+2 \partial_{+} \partial_{-} \rho+4 e^{2 \rho}\right]=0, \\
\rho: & e^{-2 \Phi}\left[2 \partial_{+} \partial_{-} \Phi-4 \partial_{+} \Phi \partial_{-} \Phi-4 e^{2 \rho}\right]=0, \\
C: & \partial_{+} \partial_{-} C=0 .
\end{array}
$$

Since we have gauge fixed $g_{++}$and $g_{--}$to zero we must also impose their equations of motion as constraints. These are

$$
\begin{gathered}
e^{-2 \Phi}\left(4 \partial_{ \pm} \rho \partial_{ \pm} \Phi-2 \partial_{ \pm}{ }^{2} \Phi\right)=-T_{ \pm \pm}^{C} \\
T_{ \pm \pm}^{C} \equiv \frac{1}{2} \partial_{ \pm} C \partial_{ \pm} C
\end{gathered}
$$

We are interested in the general infalling axion solution which is

$$
C\left(x^{+}, x^{-}\right)=C\left(x^{+}\right)
$$

Further simplifications are obtained using the residual gauge freedom to choose Kruskal gauge

$$
\Phi=\rho,
$$

so that

$$
d s^{2}=-e^{2 \Phi} d x^{+} d x^{-}
$$

In this gauge the linear dilaton vacuum is $e^{-2 \Phi}=-4 x^{+} x^{-}$with the Kruskal coordinates running over the range $-\infty<x^{-}<0,0<x^{+}<\infty$. The ++ constraint equation is

$$
\partial_{+}^{2} e^{-2 \Phi}=-T_{++}^{C}
$$

The general solution of $(2.5)$ and $(2.10)$ is then

$$
e^{-2 \Phi}=-4 x^{+} x^{-}-\int^{x^{+}} d y^{+} \int^{y^{+}} d z^{+} T_{++}^{C}\left(z^{+}\right) .
$$

Let us send in a pulse so that $T_{++}^{C}$ is non-vanishing only between $x^{+}=1$ and $x^{+}=e^{L}$. (This is an interval of length $L$ in the asymptotically inertial coordinate $\sigma^{+}=\ln x^{+}$.) The total mass of the pulse as computed on $\mathcal{I}^{-}$is

$$
M=\int d x^{+} x^{+} T_{++}^{C}
$$




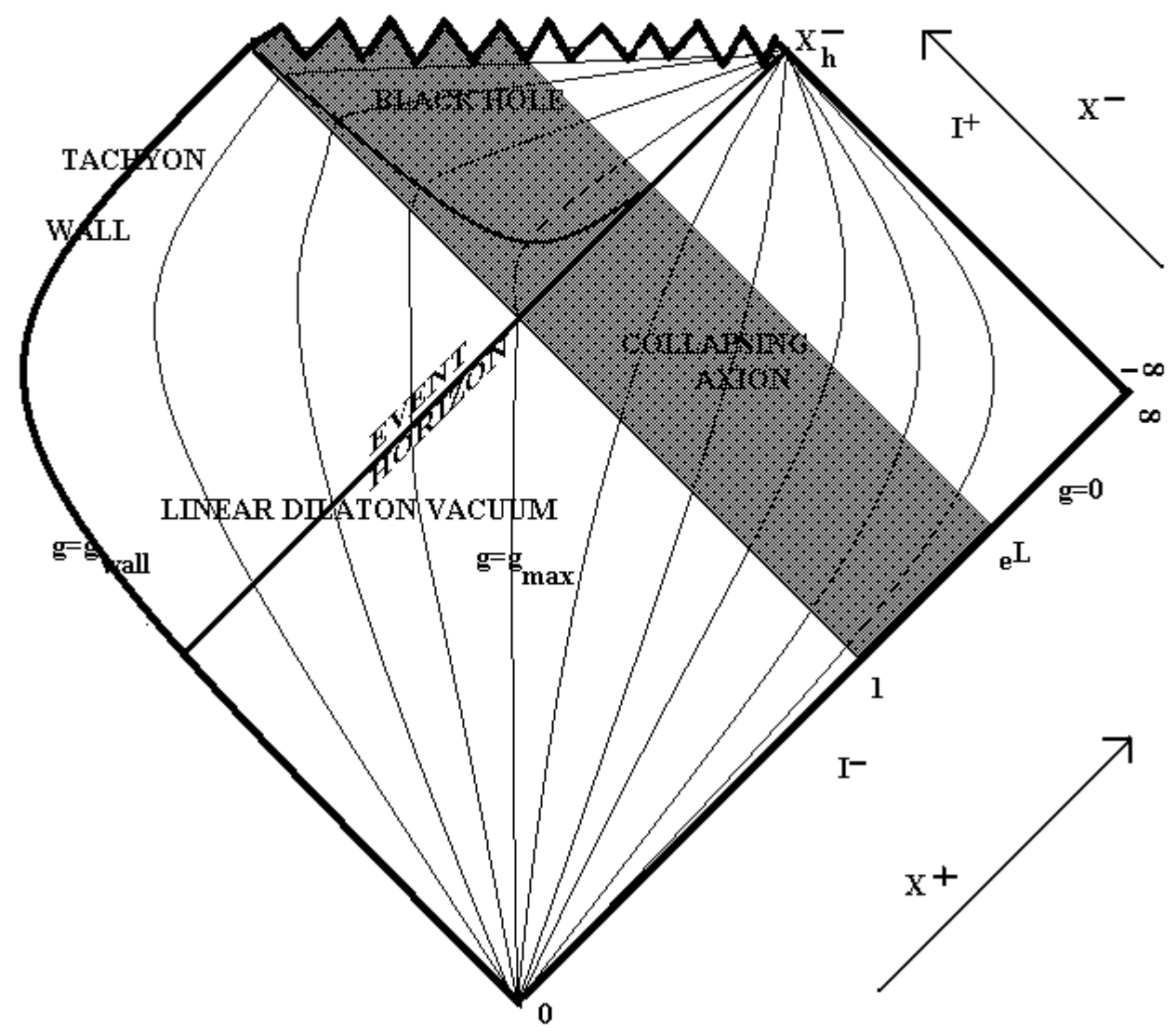

Fig. 1: Penrose Diagram for the formation of a black hole in $2 \mathrm{~d}$ string theory.

The general solution (2.11) for such a pulse is depicted in figure 1. An event horizon, defined as the boundary of the region from which light rays can escape to $\mathcal{I}^{+}$, forms at some value of $x^{-}$which we denote $x_{H}^{-}$. There is also an apparent horizon, defined as the line along which the dilaton gradient turns from spacelike to null so that $\partial_{+} e^{-2 \Phi}=0$.

3 In the derivation of (2.1) from dimensional reduction, $e^{-\Phi}$ originates as the radius of the higher dimensional spheres. Hence from this perspective the apparent horizon is the boundary of the region of trapped spheres [18]. 
Classically the apparent horizon is always inside the event horizon. It crosses the point $\left(e^{L}, x_{H}^{-}\right)$, i.e. the intersection of the event horizon with the top of the pulse. It follows that

$$
\begin{aligned}
0 & =\partial_{+} e^{-2 \Phi}\left(e^{L}, x_{H}^{-}\right) \\
& =\int_{1}^{e^{L}} d x^{+} \partial_{+}^{2} e^{-2 \Phi}\left(x^{+}, x_{H}^{-}\right)+\partial_{+} e^{-2 \Phi}\left(1, x_{H}^{-}\right) \\
& =-\int_{1}^{e^{L}} d x^{+} T_{++}^{C}\left(x^{+}\right)+\partial_{+} e^{-2 \Phi}\left(1, x_{H}^{-}\right) .
\end{aligned}
$$

Equivalently, using the fact that below the pulse we have the linear dilaton vacuum,

$$
-4 x_{H}^{-}=\int_{1}^{e^{L}} d x^{+} T_{++}^{C} .
$$

We note that the maximum value of the string coupling relevant to this computation

$$
g_{H}^{2}=-\frac{1}{4 x_{H}^{-}}
$$

occurs where the bottom of the pulse intersects the event horizon.

\subsection{Corrections}

The first issue is whether or not semiclassical perturbation theory breaks down. In order that it not break down we need the string coupling $e^{\Phi}$ to be small everywhere outside the event horizon and in the non-vacuum region above the bottom of the pulse $\left(x^{+}>1\right)$. Causalityt forbids a strong coupling region inside the horizon from affecting horizon formation. The maximum value of the string coupling in this region is given by (2.15), so in order for perturbation theory to be good we need the integral in (2.14) to be large. This is obviously easy to arrange just by making $T_{++}^{C}$ large. That is, if we throw in enough energy the black hole can be formed in a region of weak string coupling where quantum corrections to (2.1) can be made arbitrarily small.

However we still need to worry about higher-derivative $\alpha^{\prime}$ corrections to (2.1). example of such a classical term (consistent with $(2.3))$ is

$$
\int d^{2} x \sqrt{-g} e^{2 \Phi}(\nabla C)^{4} .
$$

\footnotetext{
4 Of course it is not completely clear to what extent spacetime causality follows from the matrix model.

5 For the tachyon self interaction terms such as $e^{-2 \Phi} T^{3}$ must also be considered. The absence of these for the axion facilitates the analysis.
} 
However this term identically vanishes for the solution (2.7) because we have taken $\partial_{-} C=$ 0 . To get something non-zero we need a term like

$$
\int d^{2} x \sqrt{-g} e^{2 \Phi}(\nabla C \cdot \nabla \Phi)^{4} .
$$

This would give a correction term to the stress tensor of the form

$$
\Delta T_{++}^{C} \sim e^{2 \Phi}\left(\partial_{+} C\right)^{4} .
$$

We need this to be small relative to the leading term (2.14). This is also easy to arrange. Choose constant $\partial_{+} C=f$ inside the pulse. Then we have from (2.14) and (2.15)

$$
f=\frac{\sqrt{2}}{g_{H} \sqrt{e^{L}-1}} .
$$

The correction term obeys

$$
\frac{\Delta T_{++}^{C}}{T_{++}^{C}}<2 g_{H}^{2}\left(e^{L}-1\right) f^{2}=\frac{4}{\left(e^{L}-1\right)} .
$$

So we need only take $e^{L}-1>>1$ in order for this to be a small correction. This shows that corrections of the type (2.18) cannot prevent black hole formation in all cases.

However there is another type of $\alpha^{\prime}$ correction which can not be controlled: couplings to curvature. One such term is

$$
R(\nabla C)^{2} .
$$

Since $R$ is of order one in string units at the horizon, such terms may have a large effect at the horizon. Hence we cannot be sure whether or not a black hole really forms in the full string theory.

We may also consider how the background tachyon $T$ affects the story. In the preceding discussion we have set it to zero. This is consistent with the leading equations of motion, so if there is a tachyon tadpole at some order it would be suppressed. However we could also consider the effects of the tachyon wall, i.e. a tachyon vev of the form

$$
T \sim \frac{\mu}{\sqrt{-x^{+} x^{-}}} .
$$

This produces a wall at the region where $T$ is of order one. One expects the axion to be reflected from the wall. This could be described for example by an interaction term

$$
\int d^{2} x \sqrt{-g} e^{T}(\nabla C)^{2},
$$


which effectively imposes a reflecting boundary condition for $C$ in the region where the tachyon is rapidly varying. The location of the tachyon wall can be characterized by $g_{\text {wall }}$, the value of the string coupling when $T \sim 1$. By taking

$$
g_{w a l l} \gg g_{H},
$$

we can arrange for the tachyon wall to be far behind the region where the event horizon forms. Hence all effects of the tachyon vev on events leading up to the formation of the event horizon are suppressed by powers of $\frac{g_{H}}{g_{\text {wall }}}$, which again can be made arbitrarily small. Of course if we take the tachyon wall to be outside the region where the event horizon would form, the collapsing pulse should reflect back to infinity rather than make a black hole.

In conclusion, due to uncontrollable $\alpha^{\prime}$ corrections involving the curvature near the horizon, the question of whether or not black hole formation occurs cannot be answered within low energy field theory. However, since string loop corrections can be suppressed, it is a question for classical string theory or equivalently worldsheet conformal field theory. A construction of a worldsheet CFT describing black hole formation would certainly be of great interest.

\section{Matrix model picture}

The classical limit (2.3) in spacetime corresponds to the Fermi liquid approximation [19] in the free fermion formulation of $2 \mathrm{D}$ string theory. In this section we describe the collapsing pulses of the previous section as finite perturbations of the Fermi surface and analyze their behavior. We will be working in the matrix model of the type $0 \mathrm{~B}$ string.

\subsection{Collapsing pulse as a Fermi sea perturbation}

The first step is to express the pulse in the standard linear dilaton Liouville theory coordinates

$$
t^{ \pm}=t \pm \ln \lambda
$$

where $\lambda$ is the matrix model eigenvalue governed by the Hamiltonian 6

$$
H=\frac{1}{2}\left(\partial_{t} \lambda\right)^{2}-\frac{1}{2} \lambda^{2}
$$

\footnotetext{
6 For type 0 (bosonic) theory this is $\alpha^{\prime}=\frac{1}{2}\left(\alpha^{\prime}=1\right)$.
} 
The asymptotic form of the dilaton is

$$
\Phi=-2 \ln \lambda=t^{-}-t^{+}
$$

and $d s^{2}=-d t^{+} d t^{-}$. Their relation to the Kruskal coordinates $x^{ \pm}$of the previous section is

$$
x^{ \pm}= \pm \lambda^{2} e^{ \pm 2 t}= \pm \frac{1}{2} e^{ \pm 2 t^{ \pm}}
$$

Thus, a constant $\frac{\partial}{\partial x^{+}} C=f$ becomes $\partial_{+} C=f e^{2 t^{+}}$over the region

$$
\partial_{+} C=f e^{2 t^{+}} \quad \frac{1}{2} \ln 2<t^{+}<\frac{1}{2} \ln 2+\frac{L}{2} .
$$

Notice that the pulse height reaches a maximum value

$$
\left|\partial_{+} C\right| \sim 2 f e^{L}
$$

which is exponentially large in $L$. It can be seen that this is generically the case. Actually in the matrix model it is awkward to describe pulses with $\int \partial C \neq 0$. It is not hard to arrange for this integral to vanish. In these cases one finds that the pulse reaches values of order $e^{L}$ of either sign.

According to [20,21] a RR pulse corresponds to a left-right antisymmetric fluctuation of the two branches of the Fermi sea which we denote $\eta_{R R}$. The correspondence involves the leg-pole transform

$$
\partial \eta_{R R}\left(t^{+}\right)=\int d y K(y) \partial_{+} C\left(t^{+}-y\right)
$$

where the kernel is given by

$$
K(y)=\int \frac{d k}{2 \pi}\left(\frac{\pi}{2}\right)^{-i k / 8} \frac{\Gamma(1 / 2+i k / 2)}{\Gamma(1 / 2-i k / 2)} e^{i k y}=z J_{0}(z),
$$

with

$$
z \equiv 2\left(\frac{\pi}{2}\right)^{1 / 8} e^{-y}
$$

This changes the shape of the pulse mostly on scales of order $\alpha^{\prime}$. In particular, as can be shown quite generally, the Fermi sea fluctuations corresponding to the spacetime pulses under discussion themselves have magnitude $e^{L}$ and are of either sign. As an illustration, a graph of the pulse

$$
\begin{aligned}
& \partial_{+} C=f e^{2 t^{+}} \quad \frac{1}{2} \ln 2<t^{+}<\frac{1}{2} \ln \left(e^{L}+1\right) \\
& \partial_{+} C=-f e^{2 t^{+}} \quad \frac{1}{2} \ln \left(e^{L}+1\right)<t^{+}<\frac{1}{2} \ln 2+\frac{L}{2}
\end{aligned}
$$




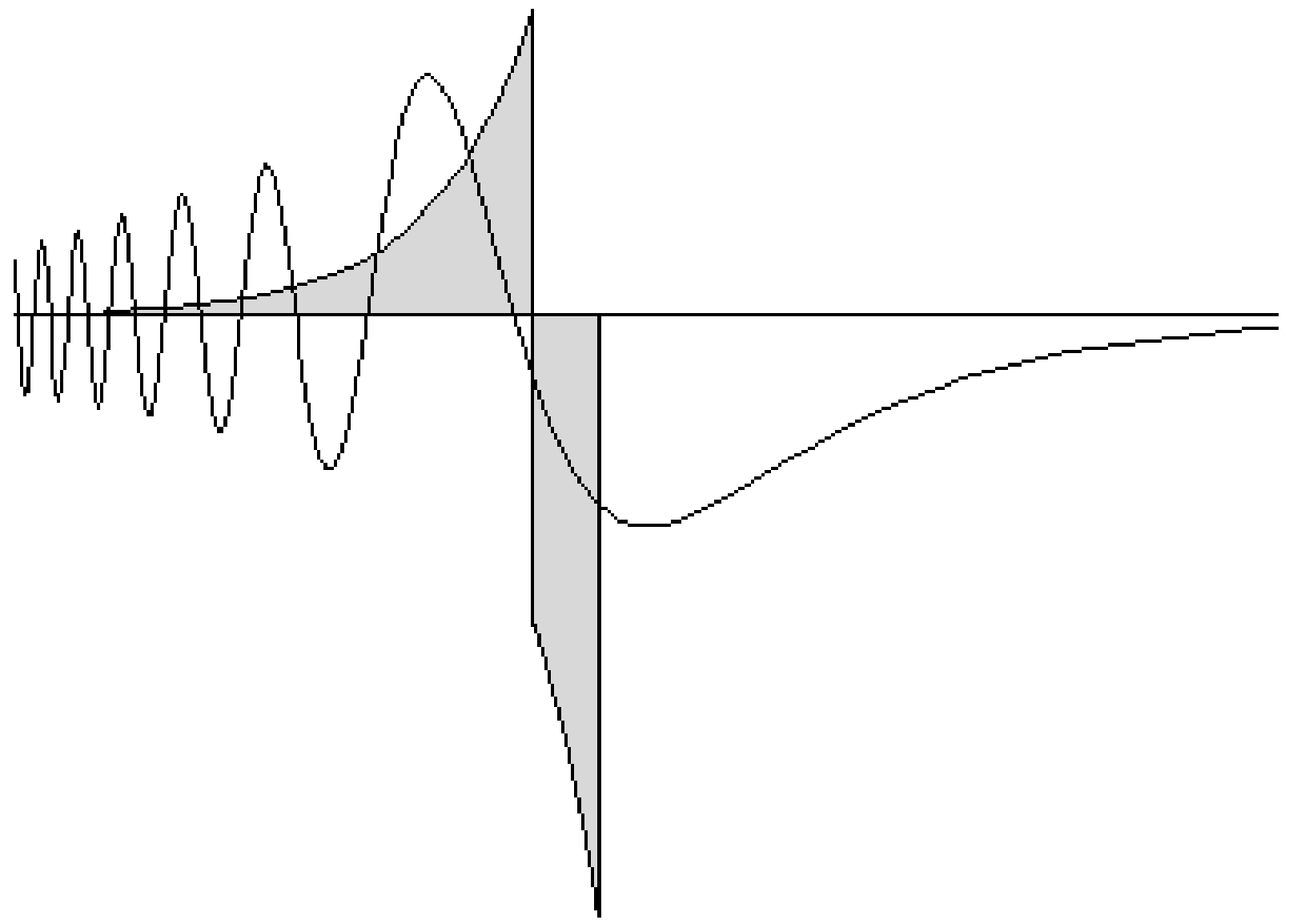

Fig. 2: Derivative $\partial_{+} C$ of the axion pulse (filled) and its leg pole transform as a function of the inertial coordinate $t^{+}$for $L=5$.

(which has $\int \partial C=0$ ), together with its leg-pole transform is given in figure 2 .

The incoming fluctuations live on the lower branch of the right hyperbola and on the upper branch of the left hyperbola. These are given by [22]

$$
p_{+}^{r}(\lambda, t)=-\sqrt{\lambda^{2}-g_{\text {wall }}^{-1}}-\frac{1}{2 \lambda} \partial_{t^{+}} \eta_{R R}
$$

and

$$
p_{+}^{l}(\lambda, t)=\sqrt{\lambda^{2}-g_{\text {wall }}^{-1}}+\frac{1}{2 \lambda} \partial_{t^{+}} \eta_{R R},
$$

which can be expanded as

$$
p_{+}^{r / l}(\lambda, t)=-\lambda+\frac{1}{2 g_{w a l l} \lambda} \mp \frac{\partial_{t^{+}} \eta_{R R}}{2 \lambda} .
$$

The pulse spills from right to left (and, correspondingly, from left to right), if at some point, $\partial_{t^{+}} \eta_{R R}>g_{\text {wall }}^{-1}\left(\partial_{t^{+}} \eta_{R R}<-g_{\text {wall }}^{-1}\right)$. From (3.6), both of these conditions are 
satisfied somewhere as long as $f e^{L}>g_{\text {wall }}^{-1}$. With (2.19), this implies $g_{H}<e^{L / 2} g_{\text {wall }}$, consistent with (2.24), the condition that the tachyon wall is well behind the region where the event horizon forms in effective theory. Note that making the pulse wider (making $L$ large) keeping $g_{H}$ fixed only makes this inequality easier to satisfy.

\subsection{Outgoing radiation on $\mathcal{I}^{+}$}

In this subsection we will see that, in the matrix model, the spilled-over peaks of the pulse (as well as the minima) are reflected off the strongly coupled region early and begin to arrive at $\mathcal{I}^{+}$at a retarded time which coincides parametrically with the time for semiclassical black hole formation. We shall further see that the majority of the pulse returns to $\mathcal{I}^{+}$in a relatively short time. We will assume that $g_{H} \ll e^{L / 2} g_{\text {wall }}$, or that the fluctuations of the incoming pulse are large compared to $g_{\text {wall }}^{-1}$.

Let the generic bottom tip of the pulse be at

$$
\partial_{t^{+}} \eta_{R R}=\epsilon \gg g_{w a l l}^{-1}
$$

This point moves along the hyperbola

$$
\lambda^{2}-p^{2}=\epsilon
$$

according to

$$
\lambda=\sqrt{\epsilon} \cosh \left(t+t_{0}\right)
$$

Using (3.1) and (3.4), we find that in the past, the point is at

$$
x^{+}=\frac{\epsilon}{8} e^{-2 t_{0}}
$$

In the future, we have

$$
x^{-}=\frac{\epsilon}{8} e^{2 t_{0}} \sim \frac{\epsilon^{2}}{x^{+}}
$$

In the past, the Kruskal coordinate of the infalling tip of the pulse is is $x^{+} \sim e^{L}$, thus, using (2.19) and (3.6), we obtain that

$$
x^{-} \sim g_{H}^{-2} \sim x_{H}^{-}
$$

A parallel computation can be performed for the spilling over peaks of the pulse, with the same conclusion. 
Ordinarily one expects that retarded times for pulse reflection diverge for $g_{\text {wall }}^{-1} \rightarrow 0$ as the tachyon wall is taken to infinity, while (3.19) is independent of $g_{w a l l}$. What is happening is that the tips of the pulse are reflected at a time determined by their energy alone. For energetic pulses obeying the condition (3.14) the value of $g_{\text {wall }}$ is not relevant. Moreover, the tip of the pulse emerges on $\mathcal{I}^{+}$where the black hole horizon is in semiclassical theory.

According to (3.18), portions of the incoming pulse on trajectories with e.g. energy $\frac{1}{2} \epsilon_{0}$ (either positive or negative) come out a time which is later by $\ln 2$ (in inertial coordinates). Hence the bulk of the pulse will return to $\mathcal{I}^{+}$over a time period which is of order one. Ignoring for a moment the effects of the leg pole transform (see below), the observer on $\mathcal{I}^{+}$ will simply see a pulse that is reflected off of the surface where the black hole would have formed in the semiclassical theory. Hence the $\alpha^{\prime}$ corrections act like a shield preventing the incoming pulse from ever getting inside its Schwarzchild radius.

The semiclassical picture is distinctly different. Hawking radiation, consisting mainly of quanta with energies of order one in string units, begins to appear on $\mathcal{I}^{+}$at retarded times of order $x_{H}^{-} \sim 1 / g_{H}$. This radiation continues until the black hole is fully evaporated, which takes a time of order the total energy $E$ of the incoming pulse, assuming a temperature and greybody factor of order one. Hence the energy return takes a parametrically longer time in this picture than it does in the exact matrix model. (See [7] for a related discussion.)

Now we show that the matrix model picture is not substantially affected by the leg pole transform. The transform (3.7) relates an outgoing collective field quantum of energy $\omega$ to that of the axion by the phase shift

$$
e^{i \omega t} \rightarrow \frac{\Gamma(1 / 2+i \omega / 2)}{\Gamma(1 / 2-i \omega / 2)} e^{i \omega t} .
$$

For $\omega$ order one in string units this implies a time delay of order the string time. For large $\omega$ we have from Stirling's formula

$$
e^{i \omega t} \rightarrow e^{i \omega(t+\ln \omega)}
$$

Hence the leg pole factor leads to a time delay of order $\ln \omega$ for highly energetic quanta. Representing the pulse (3.5) by a coherent quantum state, one finds that a typical quantum has an energy which goes at most a power of the total energy $E$. Hence the time delays will go at most like $\ln E$, and the energy is returned over a time interval which is short compared to the interval $E$ expected from the black hole picture. 


\section{Particle Production in the Spacetime Picture}

An infalling pulse of matter perturbs the quantum state of the outgoing matter, and leads to the quantum pair production of outgoing matter. There are several sources of this pair production. In this section we will compute the pair production in the spacetime picture, and in the next we will compare it to a matrix model computation. In addition to Hawking radiation, which exists for both the tachyon and the axion, we will also compute contributions due to a time dependent mass (for the tachyon) and a higher order interaction (for the axion and the tachyon). Additional outgoing particle flux arises from the $T(\partial C)^{2}$ interaction, but we will not consider those in this section, as the resulting flux has a somewhat different form from the gravitational one.

\subsection{Particle creation by an infalling pulse}

The most familiar form of pair production is the Hawking radiation due to the mismatch of time coordinates on $\mathcal{I}^{+}$and $\mathcal{I}^{-}$. This occurs for an infalling pulse even if a black hole is not formed, but is exponentially small at early retarded times. We wish to compute the leading exponential correction to the $\mathcal{I}^{+}$stress tensor for a massless particle, which may be either the axion or the tachyon, governed by the conformal gauge action

$$
\frac{1}{2 \pi} \int d^{2} u \partial_{+} S \partial_{-} S .
$$

The metric conformal factor vanishes in the far past in the coordinates

$$
t^{ \pm}= \pm \frac{1}{2} \ln \left( \pm 2 x^{ \pm}\right)
$$

and is given by

$$
\rho=\Phi+t^{+}-t^{-} .
$$

At a generic point, transforming from (2.11) one has

$$
2 \rho=-\ln \left(1-U\left(t^{+}\right) e^{2 t^{-}}\right) \sim U\left(t^{+}\right) e^{2 t^{-}},
$$

where

$$
U\left(t^{+}\right)=e^{-2 t^{+}} \int_{-\infty}^{t^{+}} d s^{+} e^{2 s^{+}} \int_{-\infty}^{s^{+}} d u^{+} e^{-2 u^{+}} T_{++}\left(u^{+}\right) .
$$

For a finite duration pulse $U$ obeys

$$
U(\infty)=\frac{1}{2} \int_{-\infty}^{\infty} d t^{+} e^{-2 t^{+}} T_{++}\left(t^{+}\right) .
$$


The asymptotic stress tensor of the Hawking particles on $\mathcal{I}^{+}$, as measured in inertial coordinates, is given by the Schwartzian of the transformation required to set $\rho=0$ on $\mathcal{I}^{+}$. This is easily seen to be, again to leading order,

$$
T_{H R--}=\frac{1}{6} U(\infty) e^{2 t^{-}} .
$$

\subsection{A time dependent mass}

In this subsection we consider the general problem of particle production by a timedependent mass term,

$$
\frac{1}{2 \pi} \int d^{2} u\left(\partial_{+} S \partial_{-} S-m^{2}\left(u^{+}, u^{-}\right) S^{2}\right),
$$

where we take $m^{2}$ to vanish on $\mathcal{I}^{ \pm}$. Such a term is forbidden for the axion but is actually present, with a form given below, for the tachyon. In general this time dependent mass will lead to particle production. We will compute the corresponding two point function in the in vacuum

$$
\Delta(s ; t) \equiv\left\langle i n\left|S\left(s^{-}\right) S\left(t^{-}\right)\right| i n\right\rangle>
$$

to first order in $m^{2}$ on $\mathcal{I}^{+}$. Expanding in powers of $m^{2}$

$$
\Delta=\Delta_{0}+\Delta_{1}+\cdots
$$

we find

$$
\frac{\partial^{2}}{\partial s^{+} \partial s^{-}} \Delta_{1}(s ; t)=-m^{2}(s) \Delta_{0}(s ; t),
$$

where

$$
\Delta_{0}(s ; t)=\ln \left(s^{-}-t^{-}\right) .
$$

There is a similar equation obtained by the interchange of $s$ and $t$. The solution of (4.11) is

$$
\Delta_{1}(s ; t)=-\int_{-\infty}^{s^{+}} d u^{+} \int_{-\infty}^{s^{-}} d u^{-} m^{2}\left(u^{+}, u^{-}\right) \Delta_{0}(u ; t)+s \leftrightarrow t,
$$

Defining the null integral of $m^{2}$

$$
m^{2}\left(u^{-}\right)=\int_{-\infty}^{\infty} d u^{+} m^{2}\left(u^{+}, u^{-}\right)
$$


(4.13) can be written on $\mathcal{I}^{+}\left(s^{+} \rightarrow \infty, t^{+} \rightarrow \infty\right)$ as

$$
\Delta_{1}\left(\infty, s^{-} ; \infty, t^{-}\right)=-\int_{-\infty}^{s^{-}} d u^{-} m^{2}\left(u^{-}\right) \ln \left(u^{-}-t^{-}\right)-\int_{-\infty}^{t^{-}} d u^{-} m^{2}\left(u^{-}\right) \ln \left(u^{-}-s^{-}\right) .
$$

We are particularly interested in the first order correction to the $\mathcal{I}^{+}$stress tensor, which is the $s \rightarrow t$ limit of the second derivative of $\Delta_{1}$

$$
T_{1--}\left(t^{-}\right)=\left.\frac{\partial^{2}}{2 \partial s^{-} \partial t^{-}} \Delta_{1}\left(\infty, s^{-} ; \infty, t^{-}\right)\right|_{s^{-}=t^{-}}=\frac{1}{2} \partial_{-} m^{2}\left(t^{-}\right) .
$$

Now we compute $m^{2}$ for the tachyon. In the linear dilaton vacuum, the wave equation for the rescaled tachyon

$$
S=e^{\Phi} T
$$

is that of a massless field. However in a background with a nontrivial metric and dilaton the tachyon acquires a mass. To leading nontrivial order in $\alpha^{\prime}$ the effective mass is

$$
m^{2}\left(u^{+}, u^{-}\right)=-4+(\nabla \Phi)^{2}-\nabla^{2} \Phi .
$$

Fixing conformal gauge and expanding

$$
\Phi=t^{-}-t^{+}+\phi
$$

one finds to linear order that

$$
m^{2}=-4\left(2 \rho-\partial_{-} \phi+\partial_{+} \phi-\partial_{+} \partial_{-} \phi\right)
$$

Imposing the gauge condition $\rho=\phi$, and using the solution (4.4) for $\phi$ and $\rho$, to leading order at early retarded times one has, 8

$$
T_{--}\left(t^{-}\right)=\frac{1}{2} \partial_{-} m^{2}\left(t^{-}\right)=4 U(\infty) e^{2 t^{-}} .
$$

Note that this has exactly the same functional dependence on the incoming stress tensor as (4.7).

In the calculation we have done, the origin of the two types of particle production seem quite different. One comes from a Bogolubov transformation on $\mathcal{I}^{+}$, while the other comes from an explicit time dependent interaction. This difference disappears in the less familiar null gauge

$$
d s^{2}=-d x^{+} d x^{-}+g_{++}\left(d x^{+}\right)^{2} .
$$

In this gauge, unlike conformal gauge, the metric approaches unity on $\mathcal{I}^{+}$and there are explicit interactions between the metric and the massless field $S$.

7 We are using here the conventional form of the effective action which does not contain for example a $T^{2} e^{-2 \Phi} \partial_{-} \partial_{+} \Phi$ term.

8 A further correction to the mass might arise if there as an $e^{-2 \Phi} R T^{2}$ term in the effective action. This would change the coefficient but not the functional form of the action. 


\subsection{The Quartic Interaction}

In this section we study an interaction of the form given in (2.16)

$$
\frac{1}{2 \pi} \int d^{2} u\left(\partial_{+} C \partial_{-} C+J e^{2 \Phi-2 \rho} \partial_{+} C \partial_{+} C \partial_{-} C \partial_{-} C\right)
$$

where $\mathrm{J}$ is a constant. Unlike the mass term in (4.8), such a term is not forbidden for the axion. There is also an identical term for the rescaled tachyon field $S$.

We are interested in how the incoming stress tensor $T_{++}$affects the outgoing $\partial_{-} c$ two point function. This is governed by the equation of motion

$$
\partial_{+} \partial_{-} C+2 J T_{++} \partial_{-}\left(e^{2 t^{-}-2 t^{+}} \partial_{-} C\right)=0
$$

where we have used (4.3). Repeating the steps of the previous section, one finds the last term in (4.24) implies an additional correction to the two-point function

$$
\Delta_{J}\left(\infty, s^{-} ; \infty, t^{-}\right)=\int_{-\infty}^{s^{-}} d u^{-} J\left(u^{-}\right)\left[\frac{2}{\left(u^{-}-t^{-}\right)}-\frac{1}{\left(u^{-}-t^{-}\right)^{2}}+\right]+t^{-} \leftrightarrow s^{-},
$$

where

$$
J\left(u^{-}\right)=-2 J e^{2 u^{-}} \int_{-\infty}^{\infty} d u^{+} e^{-2 u^{+}} T_{++}=-4 J e^{2 u^{-}} U(\infty) .
$$

The stress tensor correction is then

$$
T_{J--}\left(t^{-}\right)=\left.\frac{\partial^{2}}{\partial s^{-} \partial t^{-}} \Delta_{1}\left(\infty, s^{-} ; \infty, t^{-}\right)\right|_{s^{-}=t^{-}}=-e^{2 t^{-}} U(\infty) \frac{16 J}{3} .
$$

We note that this exactly cancels the Hawking particle production (4.7) for $J=\frac{1}{32}$.

\subsection{Particle production in string theory}

We will now perform the exact string theory computation of particle production. Note that the process we are interested in occurs in the bulk of the two dimensional spacetime, far from the Liouville wall. Such processes are fairly easy to compute in string theory, since we can simply use the linear dilaton conformal field theory. Such amplitudes were computed in [10], [11]. In order to make sense of these amplitudes one needs to consider wavepackets that are very localized, as explained in [23]. For example, we can consider localized gaussian wavepackets. We are interested in a process where an incoming leftmoving $\mathrm{RR}$ axion creates a gravitational field which in turn creates two RR axions. So the 


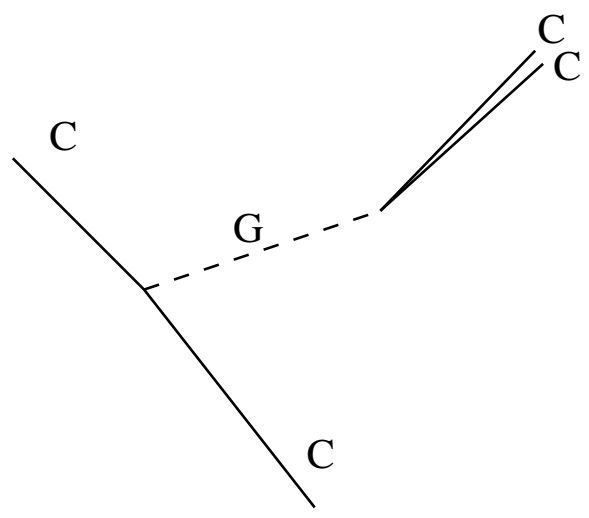

(a): $(2,2)$

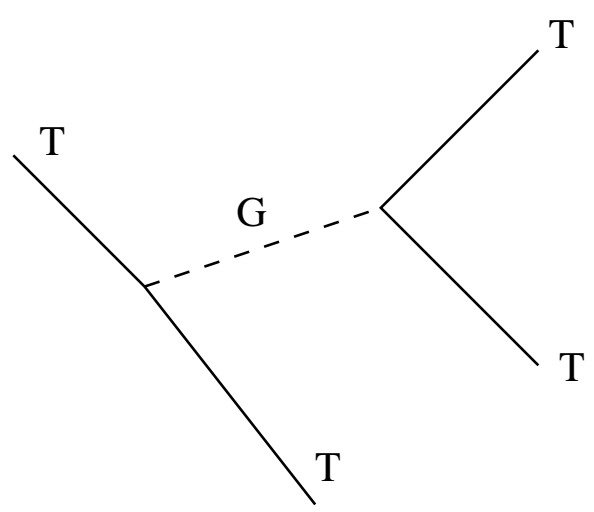

(b): $(1,3)$

Fig. 3: (a) Diagram representing creation of Hawking radiation. The graviton is not a physical propagating field, we are just thinking of it as an off shell particle. (b) Diagram used for computation of the static field created by the incoming pulse, as studied in [23]

net process is one in which we have an incoming left-moving RR axion and an out going left-moving RR axion and two right-moving RR "Hawking" particles (see figure 3(a)).

Vertex operators involve a term of the form $e^{\beta \phi-i \omega t}$. The mass shell condition is 9

$$
(\beta+2)^{2}+\omega^{2}=4 n
$$

where $n$ is an integer in the NS-NS sector and an even integer in the RR sector. The tachyon and RR axion have $n=0$, the graviton has $n=1$, etc. Massless particles have $\beta=-2 \pm i \omega$, where $+(-)$ correspond to right (left)-moving particles. Note that left-moving particles are going into the strong coupling region, while right-moving particles are going away from the strong coupling region. Once we choose the chiralities of the particles we can analytically continue in $\omega$. But we cannot analytically continue from one chirality to the other. Another important feature of the bulk amplitudes is that they preserve left-moving worldsheet fermion number $(-1)^{f_{l}}$. The tachyon and right-moving RR fields are odd and the graviton and left-moving RR field are even.

We are interested in a $(2,2)$ bulk amplitude that contains two left-moving and two right-moving particles. It was argued in [11] that the $\left(n_{l}, n_{r}\right)$ amplitude (with $n_{l}$ left movers and $n_{r}$ right movers) vanishes if $n_{r}>1$ and $n_{l}>1$. Let us see how this happens in more detail. A direct evaluation of the $(2,2)$ bulk amplitude of four RR particles gives

$$
\mathcal{A}_{\text {bulk }}=\delta\left(\sum \omega_{i}\right) \delta\left(\sum \beta_{i}+4\right) \omega_{1} \omega_{2} \omega_{3} \omega_{4} \frac{\Gamma(t / 2) \Gamma(u / 2) \Gamma\left(\frac{1}{2}+s / 2\right)}{\Gamma(1-t / 2) \Gamma(1-u / 2) \Gamma\left(\frac{1}{2}-s / 2\right)}
$$

9 We are still in units with $\alpha^{\prime}=\frac{1}{2}$. 
where we have defined

$$
\begin{aligned}
& s==-\frac{1}{4}\left[\left(\omega_{3}+\omega_{4}\right)^{2}+\left(\beta_{3}+\beta_{4}+2\right)^{2}\right] \\
& t=\text { same with } 34 \rightarrow 31, \quad u=\text { same with } 34 \rightarrow 32
\end{aligned}
$$

The $\delta$ functions in (4.29) enforce energy-momentum "conservation". The on-shell conditions for the RR axion

$$
\omega_{i}^{2}+\left(\beta_{i}+2\right)^{2}=0
$$

then imply

$$
s+t+u=1
$$

If we take particles 1 and 2 to be left-moving and particles 3 and 4 to be right-moving, then we have

$$
\beta_{1,2}=-2-i \omega_{1,2}, \quad \beta_{3,4}=-2+i \omega_{3,4}
$$

Using the on-shell conditions (4.31) one then finds

$$
s=1
$$

This in turn implies that (4.29) is zero.

We wish to understand the cancellations which lead to this result from the perspective of field theory diagrams. This can be done by relaxing the on-shell conditions (4.31), and defining the off shell amplitudes by analytic continuation in $s, t$ and $u$ in (4.29). It is convenient to do this in such a way that (4.32) is maintained. Lets first look at the $t$ channel. For $s \neq 1$ there are poles at $t=-2 n, n \geq 0$. These poles correspond to intermediate NS-NS states with $(-1)^{f_{l}}=-1$. The first pole corresponds to the tachyon and it has the simple form

$$
\mathcal{A}_{t \sim 0} \sim \frac{\omega_{1} \omega_{2} \omega_{3} \omega_{4}}{t} .
$$

This agrees with the answer we expect from the effective field theory and the coupling (2.23). Note that (4.35) does not vanish when we set $s=1$, but all higher poles do vanish when we set $s=1$. However, there is a similar pole in the $u$ channel, which has the same form as (4.35) with $t \rightarrow u$. Then (4.34) implies that $u=-t$ so that the two diagrams containing tachyon exchange vanish. This also happens in the effective field theory since we have identical kinematic restrictions.

A different situation arises if we look at the poles in the $s$ channel. Notice that the expression (4.29) has poles at $s=-1-2 n$. These poles correspond to NS-NS states with 
$(-1)^{f_{l}}=1$. The first state is the graviton. Note that the tachyon does not get exchanged since the three point coupling vanishes due to the conservation of worldsheet left fermion number 10 . In other words, the coupling between two left-moving $\mathrm{RR}$ axions and a tachyon vanishes. The pole at $s=-1$ gives a contribution of the form

$$
\mathcal{A}_{s \sim-1} \sim \frac{\omega_{1} \omega_{2} \omega_{3} \omega_{4}}{s+1}
$$

as in figure 3a. Note that when we set (4.34), (4.36) gives the contribution due to graviton exchange which can be interpreted as particle production. Since in the end the $(2,2)$ amplitude vanishes, this contribution ends up canceling after including the exchange of all higher massive states. One might imagine matching (4.29) to a low-energy action which includes only the tachyon RR axion and graviton-dilaton. In this case we would simply include the poles due to (4.35) and (4.36), then we could think about the rest as the quartic interaction of the previous subsection (the $J$ term in (4.23)) with a coefficient simply given by minus (4.36) with $s=1$.

There are other diagrams that could lead to particles on $\mathcal{I}^{+}$. For example one of the incoming (left moving) $C$ particles can scatter into a left moving tachyon and a right moving $C$ particle that goes to $\mathcal{I}^{+}$. This process can be explicitly subtracted off if we are interested in isolating the piece that comes from gravitational particle creation. In fact, we will see that the matrix model computation contains a piece that comes from this process.

Note that in [23] a rather similar diagram, involving an intermediate graviton, was computed. They sent in a pulse and then they send in a second pulse and they studied the scattering of the second pulse from the gravitational field created by the first. In this case the corresponding bulk amplitude is depicted in figure $3(\mathrm{~b})$. This is involves a $(3,1)$ bulk amplitude which does not vanish [10]1]. The authors of [23 found a detailed agreement between the effective action computations and the matrix model results, including a non vanishing $(1,3)$ amplitude. Note that the graviton which is exchanged in the case studied in [23] can be on shell. In other words the corresponding invariant is not fixed, but is a function of the external momenta.

It seems rather surprising that the effects of static gravitational fields are seen ( $23 \pi)$ but time dependent ones are delicately canceled. This might be a consequence of the $W_{\infty}$ symmetry, and it might be ultimately be the reason that black holes do not form in the singlet sector 11 .

10 In the effective field theory the couplings coming from (2.23) vanish if the two RR axions have the same chirality.

11 See [24] 25] 26] [27] for further discussion on the implications of the $W_{\infty}$ symmetry. 


\section{Particle Production in the Matrix Model}

In the previous section, we have computed particle production arising from various terms in the action. These results should be compared with the matrix model computation. The matrix model has a nontrivial S-matrix, which is further modified by the leg-pole factors and then interpreted as the worldsheet S-matrix of string theory. In principle, the various terms in the effective action which we have studied in the previous section can be computed from this information. An example of such computation was presented in [23], where the coefficients of the Einstein-dilaton term and the cubic tachyon self interaction where computed.

In the matrix model, particle production at the level at which we are studying it is related to the $1 \rightarrow 3$ S-matrix element. This should not be confused with the statement we made above, which was that the particle production came from a $(2,2)$ bulk amplitude. These two statements are consistent since one of the particles involved in the bulk computation then scatters from the wall and comes back out and becomes a right-moving particle also. The matrix model takes the full process into account. The computation we will eventually do is really an inclusive computation where we compute $1 \rightarrow 2+X$ where $X$ is anything that comes out late and the 2 represent the two right-moving particles that are created at early times. This represents an incoming particle causing the production of a particle-antiparticle pair on $\mathcal{I}^{+}$. The leading term in string coupling perturbation theory coming from the matrix model should reproduce the sum of the various terms computed in the previous section.

In [23], the computation starts out as a perturbation expansion in $\frac{1}{\mu}$ or equivalently the value of the string coupling at the tachyon wall. At the end however, we are interested

in the $\mu$-independent terms, which are a bit tricky to extract from a $\frac{1}{\mu}$ expansion. In the bosonic string considered in [23], one does not really have the option of simply working at $\mu=0$ because the theory is ill-defined there. In contrast for the $0 \mathrm{~B}$ theory there is no problem at $\mu=0$, so we will compute in the relatively simple free-fermion formalism at $\mu=0$.

\subsection{Basic formulae}

In this subsection we will summarize the basic formulae needed for the computation. 
First let us summarize the leg-pole factors.

$$
\begin{aligned}
\alpha_{\text {out }}(\omega) & =l(\omega)^{-1} \bar{\alpha}_{\text {out }}(\omega) \\
\alpha_{\text {in }}(\omega) & =l(\omega) \bar{\alpha}_{\text {in }}(\omega) \\
l(\omega)_{\text {bos }} & =\frac{\Gamma(i \omega)}{\Gamma(-i \omega)} \\
l(\omega)_{T} & =\frac{\Gamma(i \omega / 2)}{\Gamma(-i \omega / 2)} \\
l(\omega)_{C} & =\frac{\Gamma\left(\frac{1}{2}+i \omega / 2\right)}{\Gamma\left(\frac{1}{2}-i \omega / 2\right)}
\end{aligned}
$$

where $\alpha(\omega)$ is a creation operator for $\omega>0$, overbars denote matrix model fields, $T$ denotes dressed $0 \mathrm{~B}$ tachyon and $C$ denotes $\mathrm{OB}$ axion. We have chosen $\alpha^{\prime}=1$ for the bosonic string and $\alpha^{\prime}=\frac{1}{2}$ for the superstring. The $0 \mathrm{~B}$ matrix model tachyon and $\mathrm{RR}$ axion are related to the free fermions by

$$
\begin{array}{rlrl}
\psi^{R} & =e^{i(\bar{T}+\bar{C})}, & \psi^{L} & =e^{i(\bar{T}-\bar{C})} \\
\psi^{e} & =\psi^{L}+\psi^{R}, & \psi^{o} & =\psi^{R}-\psi^{L} \\
\partial \bar{T} & =\tilde{\psi}^{e} \psi^{e}+\tilde{\psi}^{o} \psi^{o}, & \partial \bar{C}=\tilde{\psi}^{e} \psi^{o}+\tilde{\psi}^{o} \psi^{e} \\
\psi^{e} & \sim e^{i \bar{T}}\left(e^{i \bar{C}}+e^{-i \bar{C}}\right), & \psi^{o} \sim e^{i \bar{T}}\left(e^{i \bar{C}}-e^{-i \bar{C}}\right)
\end{array}
$$

$\psi^{L}\left(\psi^{R}\right)$ is localized in the left (right) part of the Fermi sea, and we use $\tilde{\psi}$ to denote $\psi^{\dagger}$ to avoid a cluttering of indices. Now we consider the matrix model reflection amplitudes for even and odd fermions: $\psi^{e}, \psi^{o}$. The reflection amplitudes of fermions of energy $\epsilon$ are

$$
\begin{aligned}
\psi_{\text {in }}^{e}(\epsilon) & =i e^{-\pi i / 4} 2^{-i \epsilon} \frac{\Gamma\left(\frac{1}{4}-i \frac{\epsilon}{2}\right)}{\Gamma\left(\frac{1}{4}+i \frac{\epsilon}{2}\right)} \psi_{\text {out }}^{e}(\epsilon) \\
\psi_{\text {in }}^{o}(\epsilon) & =i e^{\pi i / 4} 2^{-i \epsilon} \frac{\Gamma\left(\frac{3}{4}-i \frac{\epsilon}{2}\right)}{\Gamma\left(\frac{3}{4}+i \frac{\epsilon}{2}\right)} \psi_{\text {out }}^{o}(\epsilon) \\
\psi_{\text {in }}^{\text {bos }}(\epsilon) & =\sqrt{\frac{\Gamma\left(\frac{1}{2}-i \epsilon\right)}{\Gamma\left(\frac{1}{2}+i \epsilon\right)}} \psi_{\text {out }}^{\text {bos }}
\end{aligned}
$$

where $\psi(\epsilon)$ is a fermion creation operator if $\epsilon>0$. We have also indicated the reflection factor in the bosonic case (this will make sense only for appropriate Fermi levels).

\subsection{Single fermion reflection}

Let us consider an incoming fermion with a wavefunction $f_{i n}(t+x)$, such that $f_{i n}(t)=$ 0 for $t<0$

$$
f_{i n}(t+x)=\frac{1}{2 \pi} \int d \omega f_{i n}(\omega) e^{-i \omega(t+x)}
$$


Then $f_{i n}(\omega)$ is analytic in the upper half plane. The out wavefunction is

$$
f_{\text {out }}(t-x)=\int d \omega R(\omega) f_{\text {in }}(\omega) e^{-i \omega(t-x)}, \quad R(\omega)=\frac{\Gamma(\kappa-i \omega / 2)}{\Gamma(\kappa+i \omega / 2)}
$$

An important property of the fermion reflection factor is that it is analytic in the upper half plane. Naively this would imply that $f_{\text {out }}(t-x)$ is zero for $t-x<0$. This is not true because $R \rightarrow \infty$ when $\omega \rightarrow i \infty$. If fact, let us consider the case where we have a Fermi level given by $\mu$ and we consider energies near the Fermi level. Then we just replace $\omega \rightarrow \mu+\omega$ in the reflection factor (5.5). For large $|\mu|$ this reflection factor has a leading phase going like $R \sim e^{-i \omega \log |\mu|}$. This means that a low energy excitation, with energy $\omega \ll|\mu|$ will lead to

$$
f_{\text {out }}(t-x) \sim f_{\text {in }}(t-x+\log |\mu|)
$$

So this looks as if the reflection is happening at $2 x=\log |\mu|$. This agrees with the intuition that the reflection occurs at $-\lambda^{2} / 2=\mu$, where $\lambda \sim e^{x}$. This is a nice check of the sign in the argument of the gamma functions (5.3).

In other words, the early time reflection that we get in the matrix model is due to the growth of $R$ in the upper half plane and not from poles in $R$. On the other hand the late time behavior of the amplitude does indeed depend on the poles in $R$.

\subsection{One point functions}

We now consider an incoming coherent state

$$
|f\rangle=e^{i \int d \omega \bar{f}(\omega) \bar{\alpha}_{i n}(\omega)}|0\rangle .
$$

where $\bar{f}^{*}(\omega)=\bar{f}(-\omega)$ is the pulse profile in the matrix model and $\bar{\alpha}_{i n}$ is a collective field oscillator.12 We want to compute

$$
\langle f|\partial \phi(t)| f\rangle \equiv\langle\partial \phi(t)\rangle_{f}
$$

12 These obey

$$
\bar{\alpha}(\omega)=i \omega \sqrt{2} \int d s e^{-i \omega s} \eta(s), \quad\left[\bar{\alpha}(\omega), \bar{\alpha}\left(\omega^{\prime}\right)\right]=2 \pi \omega^{\prime} \delta\left(\omega+\omega^{\prime}\right) .
$$


Since we are interested in early time behavior on $I^{+}$, we will simplify things a bit by taking $f$ not to have an early time tail and also obey $\int d u e^{-u} f(u)=0$. In this case the matrix model $\bar{f}$ does not contain the leading exponential piece at early times.

We can compute (5.9) by expressing the boson in terms of the matrix model fermions. For the bosonic string we obtain the expression

$$
\begin{aligned}
&\langle\partial \phi(t)\rangle_{f} \sim i \int d \omega d \epsilon e^{-i \omega t} d u d u^{\prime} e^{i(\omega / 2+\epsilon) u+i(\omega / 2-\epsilon) u^{\prime}} \\
& l(\omega) R^{-1}(-\omega / 2+\epsilon) R(\omega / 2+\epsilon) \frac{e^{i\left(\bar{f}(u)-\bar{f}\left(u^{\prime}\right)\right)}-1}{u-u^{\prime}}
\end{aligned}
$$

Where $R(\omega)$ are the fermion reflection factors (5.3) and the symbol $\sim$ here an hereafter means that factors of $2,-1$ and $\pi$ are omitted. The subtraction of -1 corresponds to normal ordering the fermions. We are interested in the early time tail, so we want to consider $t \ll 0$. In this case we may deform the $\omega$ integral to the upper half plane. Since the reflection factors are analytic in the upper half plane we see that (if we do not shift contours in $\epsilon$ ) we do not get poles from the bounce factors 13 . We do get poles from the leg factor. For the bosonic string we would get poles at $\omega=i n, n>0$, and the leading contribution comes from the first one. So we evaluate the residue of the integrand at $\omega=i$. In the bosonic string case the bounce factors give something of the form

$$
R^{-1} R \sim|\epsilon| \rightarrow|\mu|-\epsilon
$$

where the second result is the one with nonzero $\mu, \mu<0$. We now set $u=u_{c}+r / 2$, $u^{\prime}=u_{c}-r / 2$. The integrand contains a factor $e^{i \epsilon r}$, so the factor of $\epsilon$ may be converted into $i \partial_{r}$, yielding

$$
-\left.i\left(|\mu|-i \partial_{r}\right) \frac{e^{i \bar{f}\left(u_{c}+r / 2\right)-i \bar{f}\left(u_{c}-r / 2\right)}-1}{r}\right|_{r=0}=\left(|\mu| \bar{f}^{\prime}+\frac{1}{2}\left(\bar{f}^{\prime}\right)^{2}\right)
$$

In conclusion, the early time piece is proportional to

$$
\langle\partial \phi\rangle_{f} \sim e^{t} \int d u e^{u}\left(|\mu| \bar{f}^{\prime}-\frac{1}{2}\left(\bar{f}^{\prime}\right)^{2}\right)
$$

where we have set $u_{c}=u$. This answer is valid to all orders in perturbation theory. This expression is convergent if we choose $f$ so that the first tail of $\bar{f}$ vanishes. In other words,

13 Note that in this case it is OK to shift the contour to $\omega \rightarrow i \infty$ since the growth of the bounce factors is canceled by the decrease of the leg factor (away from the purely imaginary axis). 
we want $\bar{f} \ll e^{-u}$ for $u \rightarrow \infty$. In general, if we start with a completely localized $f$, then we get $\bar{f} \sim e^{-n u}$ from the leg poles. A nice derivation of this result was given in [28], by using the fact that the early time tails are given by conserved $W_{\infty}$ charges in the matrix model. Finally note that (5.13) can be interpreted as arising from a bulk interaction of the form $\int e^{-2 \Phi} T^{3}$.

We now compute similar expressions for the $0 \mathrm{~B}$ case. Let us start with the tachyon. Then we find

$$
\begin{aligned}
\langle\partial T(t)\rangle_{f}= & \int d \omega d \epsilon e^{-i \omega t} d u d u^{\prime} e^{i(\omega / 2+\epsilon) u+i(\omega / 2-\epsilon) u^{\prime}} l_{T}(\omega) \times \\
& {\left[R_{e}^{-1}(-\omega / 2+\epsilon) R_{e}(\omega / 2+\epsilon)\left\langle: \tilde{\psi}^{e}\left(u^{\prime}\right) \psi^{e}(u):\right\rangle_{f}\right.} \\
& \left.+R_{o}^{-1}(-\omega / 2+\epsilon) R_{o}(\omega / 2+\epsilon)\left\langle: \tilde{\psi}^{o}\left(u^{\prime}\right) \psi^{o}(u):\right\rangle_{f}\right]
\end{aligned}
$$

As before, we are interested in the upper half $\omega$ plane. Only the poles of the leg factor contribute. The first is at $\omega=2 i$. Then the bounce factors give

$$
R_{e}^{-1} R_{e}=R_{o}^{-1} R_{o}=\frac{1}{4}\left(\frac{1}{4}+\epsilon^{2}\right)=\frac{1}{4}\left(\frac{1}{4}-\partial_{r}^{2}\right),
$$

The first equality in (5.15) holds at all poles of the leg factor, namely it holds for $\omega=i 2 n$, $n>0$ and $n$ integer. Since the two bounce factor ratios are the same we get the combination

$$
\begin{aligned}
\left\langle: \tilde{\psi}^{e}\left(u^{\prime}\right) \psi^{e}(u):+: \tilde{\psi}^{o}\left(u^{\prime}\right) \psi^{o}(u):\right\rangle_{f} & =\left\langle: \tilde{\psi}^{L}\left(u^{\prime}\right) \psi^{L}(u):+: \tilde{\psi}^{R}\left(u^{\prime}\right) \psi^{R}(u):\right\rangle_{f} \\
& =i \frac{e^{i\left(\bar{T}(u)-\bar{T}\left(u^{\prime}\right)\right)} \cos \left(\bar{C}(u)-\bar{C}\left(u^{\prime}\right)\right)-1}{u-u^{\prime}}
\end{aligned}
$$

In this and similar expressions below, $\bar{T}$ and $\bar{C}$ to denote their coherent state expectation values in the coherent state $|\bar{f}\rangle$ rather than quantum operators. Hopefully the distinction will be clear form the context. The final result at $\mu=0$ is

$$
\langle\partial T\rangle \sim e^{2 t} \int d u e^{-2 u}\left[\bar{T}^{\prime}+4\left(\bar{C}^{\prime}\right)^{2} \bar{T}^{\prime}+\frac{4}{3}\left(\bar{T}^{\prime}\right)^{3}-\frac{1}{3} \bar{T}^{\prime \prime \prime}\right]
$$

Let us comment briefly on this result. The term involving $T^{3}$ can arise from a bulk $e^{-2 \Phi} T^{4}$ interaction. Notice that the difference between the bosonic string result and the superstring result comes from the interplay of the matrix model amplitudes and the leg factors. If we choose a different values of $\alpha^{\prime}$ in the bosonic and in the superstring so that the matrix model amplitudes are essentially the same, then the leg pole factors are different. This difference in the leg-pole factors then translate into the rather different effective actions 
in the bosonic string versus the superstring. For example the bosonic string contains a $T^{3}$ interaction which is responsible for (5.13), while no such interaction is present in the superstring. Indeed, the superstring result (5.17) is related to $T^{4}$ interactions in the bulk.

If we wanted to consider the result at non-zero $\mu$, then we must shift $\epsilon$ in (5.15) by $\mu$. Then we get terms that are at most quadratic in $\mu$.

Now let us consider the same computation for the $\mathrm{RR}$ axion

$$
\begin{aligned}
\langle\partial C(t)\rangle_{f}= & \int d \omega d \epsilon e^{-i \omega t} d u d u^{\prime} e^{i(\omega / 2+\epsilon) u+i(\omega / 2-\epsilon) u^{\prime}} l_{C}(\omega) \times \\
& {\left[R_{o}^{-1}(-\omega / 2+\epsilon) R_{e}(\omega / 2+\epsilon)\left\langle: \tilde{\psi}^{o}\left(u^{\prime}\right) \psi^{e}(u):\right\rangle\right.} \\
& \left.+R_{e}^{-1}(-\omega / 2+\epsilon) R_{o}(\omega / 2+\epsilon)\left\langle: \tilde{\psi}^{e}\left(u^{\prime}\right) \psi^{o}(u):\right\rangle_{f}\right]
\end{aligned}
$$

As before, we are interested in the upper half $\omega$ plane, and only the poles of the leg-pole factor contribute. The first one is at $\omega=i$. The bounce factors give

$$
R_{o}^{-1} R_{e}=R_{e}^{-1} R_{o}=\frac{\epsilon}{2}
$$

The first equality holds at all poles of the leg factor, namely at $\omega=i(1+2 n)$. (Note that we get basically the same as in the bosonic string (5.11)) We see that the independent factors then combine into

$$
\begin{aligned}
\left\langle: \tilde{\psi}^{e}\left(u^{\prime}\right) \psi^{o}(u):+: \tilde{\psi}^{e}\left(u^{\prime}\right) \psi^{o}(u):\right\rangle_{f}= & \left\langle: \tilde{\psi}^{R}\left(u^{\prime}\right) \psi^{R}(u):-: \tilde{\psi}^{L}\left(u^{\prime}\right) \psi^{L}(u):\right\rangle_{f}= \\
& i \frac{e^{i\left(\bar{T}(u)-\bar{T}\left(u^{\prime}\right)\right)} \sin \left(\bar{C}(u)-\bar{C}\left(u^{\prime}\right)\right)}{u-u^{\prime}}
\end{aligned}
$$

Transforming (5.19) into a derivative we find that

$$
\langle\partial C\rangle_{f} \sim e^{t} \int d u e^{-u} \partial \bar{T} \partial \bar{C}
$$

This is consistent with a bulk coupling of the form $T(\nabla C)^{2}$ (see $\left.(2.23)\right)$.

\subsection{Axion two point functions}

We now compute the connected part of the axion two point function

$$
\begin{aligned}
\langle\partial C(t) \partial C(t)\rangle_{f, c}= & \\
& \int d \omega_{1} d \omega_{1} d \epsilon_{1} d \epsilon_{2} d u_{1} d u_{2} d u_{1}^{\prime} d u_{2}^{\prime} \\
& e^{-i\left(\omega_{1}+\omega_{2}\right) t} e^{i \omega_{1}\left(u_{1}+u_{1}^{\prime}\right) / 2+i \epsilon_{1}\left(u_{1}-u_{1}^{\prime}\right)} e^{i \omega_{2}\left(u_{2}+u_{2}^{\prime}\right) / 2+i \epsilon_{2}\left(u_{2}-u_{2}^{\prime}\right)} \\
& l_{C}\left(\omega_{1}\right) l_{C}\left(\omega_{2}\right)\left\langle: \tilde{\psi}_{\text {out }}^{R}\left(u_{1}^{\prime}\right) \psi_{\text {out }}^{R}\left(u_{1}\right)-\tilde{\psi}_{\text {out }}^{L}\left(u_{1}^{\prime}\right) \psi_{\text {out }}^{L}\left(u_{1}\right):\right. \\
& \left.: \tilde{\psi}_{\text {out }}^{R}\left(u_{2}^{\prime}\right) \psi_{\text {out }}^{R}\left(u_{2}\right)-\tilde{\psi}_{\text {out }}^{L}\left(u_{2}^{\prime}\right) \psi_{\text {out }}^{L}\left(u_{2}\right):\right\rangle_{f, c}
\end{aligned}
$$


The $\psi_{\text {out }}$ operators in this expression need to be replaced by in operators using the bounce factors as above. However, we are eventually going to be interested in in setting $\omega_{1}=i$ and $\omega_{2}=i$. So in order not to get a terrible mess, we are going to evaluate just this term in the full answer. As we saw above taking $\omega_{1}=\omega_{2}=i$ will imply that the bounce factors reduce to simple terms involving $i \epsilon_{1}$ and $i \epsilon_{2}$. This, in turn become derivatives with respect to $r_{1}=u_{1}-u_{1}^{\prime}$ and $r_{2}=u_{2}-u_{2}^{\prime}$. So we get an expression of the form

$$
\begin{aligned}
\langle\partial C(t) \partial C(t)\rangle_{f, c}= & e^{2 t} \int d u_{1}^{c} d u_{2}^{c} e^{-u_{1}^{c}-u_{2}^{c}} \\
& \partial_{r_{1}} \partial_{r_{2}}\left\langle: \tilde{\psi}^{R}\left(u_{1}^{\prime}\right) \psi^{R}\left(u_{1}\right)-\tilde{\psi}^{L}\left(u_{1}^{\prime}\right) \psi^{L}\left(u_{1}\right):\right. \\
& \left.: \tilde{\psi}^{R}\left(u_{2}^{\prime}\right) \psi^{R}\left(u_{2}\right)-\tilde{\psi}^{L}\left(u_{2}^{\prime}\right) \psi^{L}\left(u_{2}\right):\right\rangle\left._{f, c}\right|_{r_{i}=0}
\end{aligned}
$$

After taking derivatives, this expectation value is proportional to

$$
\frac{1}{\left(u_{1}^{c}-u_{2}^{c}\right)^{2}} \partial \bar{C}\left(u_{1}^{c}\right) \partial \bar{C}\left(u_{2}^{c}\right)
$$

where for simplicity we have taken an antisymmetric incoming pulse with $\bar{T}=0$.

The final answer is

$$
\langle: \partial C(t) \partial C(t):\rangle_{f} \sim e^{2 t} \int d u_{1} d u_{2} e^{-u_{1}-u_{2}} \frac{1}{\left(u_{1}-u_{2}\right)^{2}} \partial \bar{C}\left(u_{1}\right) \partial \bar{C}\left(u_{2}\right)
$$

In treating the double pole we note that it arises from the contraction

$$
\psi^{*}\left(u_{1}\right) \psi\left(u_{2}\right)-: \psi^{*}\left(u_{1}\right) \psi\left(u_{2}\right): \sim \frac{i}{u_{1}-u_{2}+i \epsilon} \sim \psi\left(u_{1}\right) \psi^{*}\left(u_{2}\right)-: \psi\left(u_{1}\right) \psi^{*}\left(u_{2}\right):
$$

Since the denominator in (5.25) came from performing these contractions we should replace it by $1 /\left(u_{1}-u_{2}+i \epsilon\right)^{2}$. It now easy to see that there is no divergence.

We see that (5.25) does not have the interpretation of the Hawking radiation that we were expecting. Nevertheless we would like to identify the spacetime origin of (5.25). In the spacetime action we have a coupling of the form (2.23)

$$
S_{i n t} \sim \int e^{\Phi} T(\partial C)^{2}
$$

This is the same coupling that was responsible for the one point function (5.21). This coupling can lead to a process where one incoming $\mathrm{RR}$ axion becomes an ingoing tachyon and an outgoing RR axion. By summing over the tachyon final states, then we can get (5.25). In fact the whole answer is accounted for in this fashion. This implies that all other processes cancel. In fact, this is consistent with the fact that the $(2,2)$ bulk scattering string amplitude is zero. 


\subsection{Tachyon two point function}

The situation is rather different for the two point function of the $0 \mathrm{~B}$ tachyon on $\mathcal{I}^{+}$. For the axion, the exponential tail was produced by the leg pole transform. The falloff rate had the right strength to correspond to Hawking radiation but, as we saw, the detailed functional form indicated an alternate source. For the tachyon, the leg pole transform falls of at twice the rate, so that

$$
\langle\partial T(t) \partial T(t)\rangle_{f} \sim e^{4 t}
$$

Hence there is no hope of getting anything large enough to correspond to the rising tail of Hawking radiation. As mentioned before, this is not a contradiction because the leading tail of Hawking radiation can be canceled by other interactions if they have certain finelytuned coefficients. In fact the bulk scattering amplitude to 2 RR fields into 2 tachyons or 2 tachyons into two 2 tachyons vanishes [11].

\section{Scattering amplitudes for very energetic states}

In this section we consider a single incoming tachyon in the type 0A theory with very large energy $\epsilon \gg 1$. For simplicity we set $\mu=0$. We are then interested in seeing if there is some sign of black hole formation. Specifically we will compute the number of particles with energies $\omega$ that we have in the final state. This computation is very similar to those in [6] [7]. In particular, the analysis of [7] is more detailed than what we are going to perform here. We decided to include this because is it is a rather simple and direct computation.

The exact fermion scattering amplitude is given by 14

$$
R(\epsilon)=\frac{\Gamma\left(\frac{1}{2}-i \frac{\epsilon}{2}\right)}{\Gamma\left(\frac{1}{2}+i \frac{\epsilon}{2}\right)}
$$

An incoming boson with energy $\epsilon$ may be written as

$$
|\Psi\rangle=\alpha_{i n}(-\epsilon)|0\rangle=\int_{0}^{\epsilon} d x \psi_{i n}^{\dagger}(x) \psi_{i n}(\epsilon-x)|0\rangle
$$

In terms of out operators

$$
|\Psi\rangle \equiv \int_{0}^{\epsilon} d x R^{*}(-x) R(\epsilon-x) \psi_{\text {out }}^{\dagger}(x) \psi_{\text {out }}(\epsilon-x)|0\rangle
$$

14 Up to phases that are constant or linear in the energy. Such terms do no matter for this computation. 
We can compute the norm of (6.3) and we find that it is equal to

$$
\langle\Psi \mid \Psi\rangle=\epsilon \delta(0)
$$

where the $\delta(0)$ is just the usual volume factor due to the fact that we are considering plane waves. Now we want to compute the expectation value for the number of particles with fixed energy $\omega$ in the outgoing state $(6.3)$. Namely we are interested in computing

$$
\omega N_{\omega}=\left\langle\Psi\left|\alpha_{\text {out }}(-\omega) \alpha_{\text {out }}(\omega)\right| \Psi\right\rangle
$$

Note that leg pole factors cancel out in this computation. In order to obtain $N_{\omega}$ we first compute that

$$
\begin{aligned}
\alpha_{\text {out }}(\omega)|\Psi\rangle & =\int_{\omega}^{\epsilon} d x F(x) \psi_{\text {out }}^{\dagger}(x-\omega) \psi_{\text {out }}(\epsilon-x)|0\rangle \\
F(x) & =R(-x)^{*} R(\epsilon-x)-R^{*}(-(x-\omega)) R(\epsilon-x+\omega)
\end{aligned}
$$

where we assumed that $\omega \neq \epsilon$ (otherwise there is an additional term). Note $\alpha_{0}$ annihilates the state $(6.2)$, since $\alpha_{0}$ is the fermion number operator and the net fermion number of (6.2) is zero.

Now we go back to (6.5) which is equal to the norm of the state $(6.6)$. We find that (6.5) becomes (up to a $\delta(0)$ )

$$
\begin{aligned}
& \int_{\omega}^{\epsilon} d x\left|R^{*}(-x) R(\epsilon-x)-R^{*}(-(x-\omega)) R(\epsilon-x+\omega)\right|^{2}= \\
& \int_{\omega}^{\epsilon} d x\left|1-R(-x) R^{*}(\epsilon-x) R^{*}(-(x-\omega)) R(\epsilon-x+\omega)\right|^{2}= \\
& \int_{\omega}^{\epsilon} d x\left(2-2 R e\left(R R^{*} R^{*} R\right)\right)
\end{aligned}
$$

where we used that $|R|=1$. Using Stirling's formula we can show that the leading phase at large $x$ is

$$
\frac{\Gamma\left(\frac{1}{2}-i \frac{x}{2}\right)}{\Gamma\left(\frac{1}{2}+i \frac{x}{2}\right)} \sim e^{-i x \log x}
$$

We then see that for large $x$

$$
R(-x) R^{*}(-(x-\omega)) \sim e^{i \omega \log x}
$$

Similarly we obtain that for large $\epsilon-x$

$$
R^{*}(\epsilon-x) R(\epsilon-x+\omega) \sim e^{-i \omega \log (\epsilon-x)}
$$


We conclude that the product of all phases is

$$
x^{i \omega}(\epsilon-x)^{-i \omega}=t^{i \omega}(1-t)^{-i \omega}, \quad \text { where } \quad t=\frac{x}{\epsilon}
$$

We now substitute (6.11) in the last term of the integral (6.7) and change the integration variable to $t$. We need to evaluate an integral of the form

$$
\int_{\omega / \epsilon}^{1} d t t^{i \omega}(1-t)^{-i \omega}=\Gamma(1-i \omega) \Gamma(1+i \omega)+o(\omega / \epsilon) \sim \frac{\pi \omega}{\sinh \pi \omega} .
$$

Putting all this together we find that (6.5) has the form

$$
\left\langle\omega N_{\omega}\right\rangle=\frac{\left\langle\Psi\left|\alpha_{\text {out }}(-\omega) \alpha_{\text {out }}(\omega)\right| \Psi\right\rangle}{\langle\Psi \mid \Psi\rangle}=2\left(1-\frac{\pi \omega}{\sinh \pi \omega}\right), \quad \text { for } \omega \ll \epsilon
$$

Notice that this result goes to zero for $\omega \rightarrow 0$ as expected.

The expectation value (6.13) goes to a constant for large $\omega$. If the physical process were black hole formation we would have expected a thermal factor with a temperature of order one. We might imagine that the first, constant, factor represents a reflected pulse that arises at the moment we form a black hole and that the second factor is proportional to Hawking radiation. Indeed the second factor has an exponential decay reminiscent of Hawking radiation. But the second factor has the wrong sign and its integral is independent

of $\epsilon$ 15. The expectation value $(\underline{6.13})$ is saying that we have an energy of order one in each mode of frequency $\omega$ (for $1 \ll \omega \ll \epsilon$ ). Hence there is no indication from this computation that a highly energetic tachyon forms a black hole. Similar observations were made in [6,7].

\section{Discussion}

We close with a discussion of several issues concerning black holes in the matrix model in the context of our results.

15 By writing $\frac{1}{\sinh \pi \omega} \sim e^{-\pi \omega} \sim e^{-\beta \omega}$ for large $\omega$ in 6.13 we can read off the a temperature temperature which is the same as the temperature of the $0 \mathrm{~A} \mathrm{SL}(2) / \mathrm{U}(1)$ black hole, $\beta=2 \pi \sqrt{\alpha^{\prime} / 2}$. 


\subsection{The definition of time}

One issue concerns the time coordinate to be used in the spacetime/matrix model dictionary. In spacetime computations, there are several natural retarded time coordinates on $\mathcal{I}^{+}$. One of them is where the conformal factor $\rho$ asymptotically vanishes and the linear dilaton is time-independent. We refer to these as inertial coordinates, as they are associated to asymptotically inertial observers. The null coordinates

$$
d s^{2}=-d t_{\text {inertial }}^{+} d t_{\text {inertial }}^{-}+g_{++}\left(d t_{\text {inertial }}^{+}\right)^{2}
$$

can be seen to be inertial in this sense.

A more common coordinate choice (employed for example in this paper and [23]) is conformal gauge

$$
d s^{2}=-e^{2 \rho} d t_{\text {conformal }}^{+} d t_{\text {conformal }}^{-} .
$$

The conformal retarded time and inertial retarded times are related at early times on $\mathcal{I}^{+}$ via

$$
t_{\text {conformal }}^{-}=t_{\text {inertial }}^{-}-\frac{1}{2} U(\infty) e^{2 t_{\text {inertial }}^{-}}+\cdots
$$

These two coordinates systems define outgoing vacua on $\mathcal{I}^{+}$which are related by a nontrivial Bogolubov transformation. If we work in the conformal gauge, Hawking particle production is seen indirectly as a consequence of this Bogolubov transformation to inertial coordinates. In the inertial coordinates (7.1), particle production arises directly from the coupling of the time-dependent metric component $g_{++}$to the massless field $S$.

In order to compare the matrix model with the spacetime results we must know whether the matrix model result corresponds to conformal, inertial, or yet another coordinate choice. Although the literature is not very explicit on this point, in most discussions of the matrix model it is implicitly assumed that the matrix model time $t_{m m}$ and eigenvalue are related to the spacetime retarded time via

$$
t_{m m}-\ln \lambda=t_{\text {inertial }}^{-}
$$

This assumption has been adopted in this paper in equation (3.1), and we have seen herein that the full string theory answer is consistent with the matrix model answer only when we make this (standard) assumption.

In this paper we are considering normalizable excitations of the ground state. In the context of large, non-normalizable perturbations, it is less obvious how time should be defined. Some discussion is in [13,14, 16]. 


\subsection{The non-singlet sector}

In this paper we have analyzed whether black holes could form in the singlet sector. Critics have pointed out that our efforts were doomed to fail because it is known that the $S L(2) / U(1)$ black hole involves non singlets. However, it is also known that the $S L(2) / U(1)$ solution is not the only one that looks like a black hole 16 . So it might well be that there are other solutions, which exist purely in the singlet sector, which also have a black hole interpretation, even if they are not the standard $S L(2) / U(1)$ black hole. Our results, however, are consistent with the idea that there are no black holes in the singlet sector. So a plausible explanation for the peculiar horizon-aversion of collapsing shells found in section 3 is that a black hole cannot be formed because the matter is in the singlet sector and the black hole is not.

If the non-singlet nature of black holes is the explanation, one may also ask if black holes can be formed from non-singlet initial data. For example one might try to form them by collapsing D0-branes, which also can be argued to involve non-singlets. It would also be nice to know what the matrix model dual to a Lorentzian black hole is, as opposed to euclidean black hole [5].

\section{3. $S L(2)_{k} / U(1)=$ black hole for small $k$ ?}

For large $k$, Witten's $S L(2)_{k} / U(1)$ coset is a weakly coupled sigma model describing a black hole. For small $k$ the sigma model becomes strongly coupled so it is no longer clear if it should be thought of as a black hole. In the matrix model context, the relevant cosets always are in the small $k$ regime. Therefore it is not clear if they should be interpreted as black holes, as we discuss in this section.

As it was discussed in 29] the nature of the $S L(2)_{k} / U(1)$ conformal field theory depends in an important way on $k$. For large $k, k>3$, this conformal field theory has a normalizable zero mode which corresponds to changing the value of the dilaton at the tip 17 . For $k \leq 3$ this mode becomes non-normalizable. This can be seen from the algebraic analysis of the $S L(2)_{k} / U(1)$ model as follows. The zero mode is constructed from discrete representations as $J_{-1}^{-} \bar{J}_{-1}^{-}|j, m\rangle$ with $j=m=18$. This is a state with worldsheet

16 There are configurations that look like black holes with different radii [5].

17 Actually, the theory has two zero modes one changes the value of the dilaton and the other changes the integral of $B_{N S}$ on the cigar.

18 In conventions as in [30]. See [31] for further discussions about this state. 
conformal weight one. In general, a state is normalizable only if $\frac{1}{2}<j<\frac{k}{2}-\frac{1}{2}$, so this state with $j=1$ is normalizable only for $k>3$. From the target space point of view this seems surprising. It is clear that the wavefunction of the vertex operator that changes the dilaton at the tip decays as $e^{-2 \phi}$ for $\phi \rightarrow \infty$ while the normalizability condition only requires that it decays faster than $e^{-\phi}$ 19. The crucial point is that a mode on the cigar does not have definite winding at infinity. A good way to think about it is to imagine that the wavefunction of a given mode contains all possible winding numbers. In particular, the wavefunction of this mode goes as

$$
V_{\delta \Phi_{0}} \sim J_{-1}^{-} \bar{J}_{-1}^{-} e^{-2 \phi}+e^{-2(k / 2-1) \phi} e^{+i \sqrt{k}\left(X_{L}-X_{R}\right)}+e^{-2(k / 2-1) \phi} e^{-i \sqrt{k}\left(X_{L}-X_{R}\right)}+\cdots
$$

where the dots indicate higher winding modes. We see that the winding number one terms in (7.5) are not normalizable for $k \leq 3$.

The conclusion from these remarks is that the cigar conformal field theory at $k \leq 3$ does not have a zero mode and furthermore, when we go to the weakly coupled region, it differs from the linear dilaton theory by a non-normalizable operator. This operator is the one appearing in the second term in (7.5). Note that we have derived all this from analysis purely in the $S L(2, R)_{k} / U(1)$ theory. It is not necessary to assume that there is a dual sine-Liouville theory. Of course, the fact that the operator is not-normalizable is clear in the sine-Liouville theory and in a different context it was noted in [32]. Even though this can be derived purely from the $S L(2, R)_{k} / U(1) \mathrm{CFT}$, these facts cannot be derived from the lowest order in $\alpha^{\prime}$ analysis of the sigma model, even if we use the supposedly "exact" metrics in the literature. Any analysis that focuses only on the lowest order in $\alpha^{\prime}$ will miss the winding modes, which are not included. The point is that the curvatures are large and it is necessary to solve the theory exactly in $\alpha^{\prime}$.

For $k>3$ the existence of this zero mode is conceptually related to the fact that the asymptotic value of the radius of the cigar cannot be changed, any attempt to change the value of the radius will drive the value of the dilaton at the tip to zero or infinity. On the other hand, for $k<3$ there is no zero mode, so we expect that it should be possible to change the value of the radius. Indeed, the matrix model analysis of [5] shows that the value of the radius can be changed.

19 The last statement is saying how we are normalizing $\phi$. 
All of these points are highlighting the fact that the Lorentzian continuation of the cigar conformal field theory is not obvious. In particular, we would need to understand the Lorentzian interpretation of the extra winding mode operator that is being turned on 20 .

One can, nevertheless, be very naive and compute the absorption cross section for the black hole. If the absorption is nonzero it is reasonable to call the geometry a black hole. For this one can compute the momentum two point function in the cigar geometry and analytically continue the result to Lorentzian signature. This gives a non-zero answer for the absorption [33] [34], even when $k \leq 3$. If we believe this continuation it can be taken as evidence that the $S L(2, R)_{k} / U(1) \mathrm{CFT}$ is a black hole even for $k \leq 3$.

\subsection{Conclusions}

Previous investigations of high energy tachyon scattering [6] [7], have shown that it does not lead to the formation of black holes. In those studies scattering of a single high energy tachyon was considered. In this paper we considered the collapse of a coherent state formed by many tachyons. By looking at the time at which the energy is emitted we concluded that there cannot be a long lived black hole. The energy is emitted within a time of order $\log E$ of the the time at which the black hole would be formed. If a black hole had formed we would have expected that a finite fraction of the incoming energy would come out over a time proportional to $E$. This simple argument does not rule out the formation of a black hole with very high temperature.

On a different, but related note, we have also asked here the following question: to what extent is the low energy effective action corrected? We know from the analysis of [23], that many of its features are indeed consistent with the matrix model. In particular, [23] have shown that the matrix model includes gravitational interactions which are present in the linear dilaton theory, in the region far from the Liouville wall. Particle creation is another effect mediated by the gravitational field, so our naive expectation was that we were going to see it if we repeated the analysis of [23]. However, here there is an interesting cancellation between the gravitational effect and the effects coming from the exchange of all massive fields. This cancellation, which is present in the exact string theory answer, implies that there is no net particle creation by an infalling pulse.

20 A naive Lorentzian's continuation of the matrix model in [5] is not possible because it would lead to a Lagrangian that is not local in time, since the Wilson lines are in the exponent. Maybe a local Hamiltonian might be obtained after "integrating in" some quark degrees of freedom... 
Our computation of particle creation by the infalling pulse focused just on the leading term. It would be nice to see what happens at higher orders in the $e^{t}$ expansion.

\section{Acknowledgments}

We are grateful to I. Klebanov, F. Larsen, J. Polchinski, S. Shenker, N. Seiberg, T. Takayanagi and H. Verlinde for useful discussions. This work was supported in part by DOE grant DE-FG02-91ER40654 and the Harvard Society of Fellows. 


\section{References}

[1] E. Witten, "On string theory and black holes," Phys. Rev. D 44, 314 (1991).

[2] S. Elitzur, A. Forge and E. Rabinovici, "Some global aspects of string compactifications," Nucl. Phys. B 359, 581 (1991).

[3] G. Mandal, A. M. Sengupta and S. R. Wadia, "Classical solutions of two-dimensional string theory," Mod. Phys. Lett. A 6, 1685 (1991).

[4] S. B. Giddings and A. Strominger, "Exact black five-branes in critical superstring theory," Phys. Rev. Lett. 67, 2930 (1991).

[5] V. Kazakov, I. K. Kostov and D. Kutasov, 'A matrix model for the two-dimensional black hole," Nucl. Phys. B 622, 141 (2002) arXiv:hep-th/0101011.

[6] G. W. Moore and R. Plesser, "Classical scattering in (1+1)-dimensional string theory," Phys. Rev. D 46, 1730 (1992) arXiv:hep-th/9203060.

[7] E. Martinec and K. Okuyama, "Scattered results in 2D string theory," arXiv:hepth/0407136.

[8] J. Polchinski, "What is string theory?," arXiv:hep-th/9411028.

[9] S. Shenker, unpublished.

[10] D. J. Gross and I. R. Klebanov, "S = 1 for c $=1$," Nucl. Phys. B 359, 3 (1991).

[11] P. Di Francesco and D. Kutasov, "World sheet and space-time physics in twodimensional (Super)string theory," Nucl. Phys. B 375, 119 (1992) arXiv:hepth/9109005].

[12] J. J. Friess and H. Verlinde, "Hawking effect in 2-D string theory," arXiv:hepth/0411100.

[13] J. L. Karczmarek and A. Strominger, "Closed string tachyon condensation at c $=1$," JHEP 0405, 062 (2004) [arXiv:hep-th/0403169.

[14] S. R. Das, J. L. Davis, F. Larsen and P. Mukhopadhyay, "Particle production in matrix cosmology," Phys. Rev. D 70, 044017 (2004) arXiv:hep-th/0403275].

[15] P. Mukhopadhyay, JHEP 0408, 032 (2004) arXiv:hep-th/0406029.

[16] J. L. Karczmarek, A. Maloney and A. Strominger, "Hartle-Hawking vacuum for c = 1 tachyon condensation," arXiv:hep-th/0405092.

[17] C.G. Callan, S.B. Giddings, J.A. Harvey, and A. Strominger, Phys. Rev. D45 (92) R1005.

[18] J.G. Russo, L. Susskind, and L. Thorlacius, Phys. Lett. B292 (92) 13.

[19] J. Polchinski, "Classical Limit Of (1+1)-Dimensional String Theory," Nucl. Phys. B 362, 125 (1991).

[20] T. Takayanagi and N. Toumbas, "A matrix model dual of type $0 \mathrm{~B}$ string theory in two dimensions," JHEP 0307, 064 (2003) arXiv:hep-th/0307083.

[21] M. R. Douglas, I. R. Klebanov, D. Kutasov, J. Maldacena, E. Martinec and N. Seiberg, "A new hat for the c $=1$ matrix model," arXiv:hep-th/0307195. 
[22] S. Gukov, T. Takayanagi and N. Toumbas, "Flux backgrounds in 2D string theory," JHEP 0403, 017 (2004) [arXiv:hep-th/0312208.

[23] M. Natsuume and J. Polchinski, "Gravitational Scattering In The $\mathrm{C}=1$ Matrix Model," Nucl. Phys. B 424, 137 (1994) arXiv:hep-th/9402156].

[24] I. R. Klebanov, "Ward identities in two-dimensional string theory," Mod. Phys. Lett. A 7, 723 (1992) [arXiv:hep-th/9201005].

[25] D. Kutasov, E. J. Martinec and N. Seiberg, "Ground rings and their modules in 2-D gravity with c $\leq 1$ matter," Phys. Lett. B 276, 437 (1992) arXiv:hep-th/9111048.

[26] S. R. Das, A. Dhar, G. Mandal and S. R. Wadia, "W infinity ward identities and correlation functions in the C = 1 matrix model," Mod. Phys. Lett. A 7, 937 (1992) [Erratum-ibid. A 7, 2245 (1992)] |arXiv:hep-th/9112052.

[27] U. H. Danielsson, "Symmetries and special states in two-dimensional string theory," Nucl. Phys. B 380, 83 (1992) arXiv:hep-th/9112061.

[28] J. Polchinski, "On the nonperturbative consistency of $\mathrm{d}=2$ string theory," Phys. Rev. Lett. 74, 638 (1995) arXiv:hep-th/9409168.

[29] J. Maldacena, talk at strings 2004.

[30] J. M. Maldacena and H. Ooguri, J. Math. Phys. 42, 2929 (2001) arXiv:hepth/0001053].

[31] D. Kutasov and N. Seiberg, JHEP 9904, 008 (1999) arXiv:hep-th/9903219.

[32] D. Kutasov and N. Seiberg, "Noncritical Superstrings," Phys. Lett. B 251, 67 (1990).

[33] A. Giveon, A. Konechny, A. Pakman and A. Sever, "Type 0 strings in a 2-d black hole," JHEP 0310, 025 (2003) arXiv:hep-th/0309056.

[34] R. Dijkgraaf, H. Verlinde and E. Verlinde, "String propagation in a black hole geometry," Nucl. Phys. B 371, 269 (1992). 